\title{
Luteinizing hormone compromises the in vivo anti-tumor effect of cisplatin on human epithelial ovarian cancer cells
}

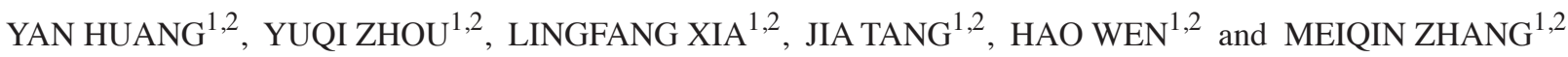 \\ ${ }^{1}$ Department of Gynecologic Oncology, Fudan University Shanghai Cancer Center; \\ ${ }^{2}$ Department of Oncology, Shanghai Medical College, Fudan University, Shanghai 200032, P.R. China
}

Received July 6, 2016; Accepted November 2, 2017

DOI: $10.3892 / \mathrm{ol} .2017 .7655$

\begin{abstract}
Platinum-based chemotherapy is the most common therapeutic regimen used to treat patients with ovarian cancer. However, the emergence of drug resistance to platinum compromises the clinical success of this treatment. Epithelial ovarian cancer is usually accompanied by an increased level of luteinizing hormone ( $\mathrm{LH})$. Therefore, the effect of $\mathrm{LH}$ on platinum resistance requires further investigation. In the current study, the effect of cisplatin and/or LH on platinum resistance was examined using the SKOV3ip1 and HeyA8 models. Following therapy, tumors were examined for proliferation (ki67) and apoptosis (cleaved caspase-3). Cisplatin alone and in combination with LH significantly inhibited tumor growth in SKOV3ip1- and HeyA8-implanted mice. Treatment with LH alone had minimal effect in the models. However, treatment with cisplatin combined with LH was less effective than treatment with cisplatin alone. Additionally, ki67 counts were significantly increased and cleaved caspase-3 counts were significantly reduced in mice treated with cisplatin combined with LH compared with mice treated with cisplatin alone. Such results indicate that $\mathrm{LH}$ weakens the anti-tumor effect of cisplatin in vivo and that LH may contribute to the development of drug resistance to cisplatin in ovarian cancer.
\end{abstract}

\section{Introduction}

Ovarian cancer is often diagnosed when it is at an advanced stage. The overall 5 -year survival rate of patients with ovarian cancer is $\sim 40 \%$ (1) and ovarian cancer is one of the leading causes of gynecological cancer-related mortality. The exact cause of epithelial ovarian cancer has not yet been determined. It has been suggested that gonadotropins, including follicle-stimulating hormone (FSH) and luteinizing hormone

Correspondence to: Dr Meiqin Zhang, Department of Gynecologic Oncology, Fudan University Shanghai Cancer Center, 270 Dongan Road, Shanghai 200032, P.R. China

E-mail: zhangmq67@126.com

Key words: ovarian cancer, cisplatin, luteinizing hormone, in vivo
(LH) serve an important role in the development of ovarian cancer (2), although their underlying mechanisms of action remain unknown (3). Ovarian epithelial cancer is usually characterized by elevated levels of FSH and LH, particularly in post-menopausal women or in women receiving treatment to induce ovulation (4-7). Furthermore, the results of epidemiological studies have revealed that reduced exposure to, or lower levels of gonadotropins are associated with a decreased risk of ovarian cancer (4-7). Lower levels of gonadotropins may be induced following multiple pregnancies, breast-feeding, the use of oral contraceptives and during estrogen replacement therapy $(8,9)$. Compared with FSH, the effect of LH on ovarian cancer is contentious. It has been reported that there is no association between LH and ovarian cancer cell proliferation (10); furthermore, studies have demonstrated that LH may inhibit or stimulate the progression of ovarian cancer (11-16).

Cisplatin has been widely used to treat various solid malignancies, including ovarian cancer, with a consistent rate of initial responses (17). Following the binding of cisplatin to DNA, unrepairable DNA lesions are generated, the DNA damage response is activated and mitochondrial apoptosis or proliferative arrest is subsequently induced (17). However, resistance to cisplatin readily develops and this may compromise its anti-tumor effect, resulting in therapeutic failure (17). Therefore, it is important to understand the underlying mechanisms of cisplatin resistance. It has been demonstrated that the development of cisplatin resistance is a complicated process that arises at diverse stages of DNA-targeting (17).

LH may inhibit cisplatin-induced apoptosis in vitro (18). Therefore, the present study used epithelial ovarian cancer cells to determine whether LH impairs the in vivo anti-tumor effect of cisplatin in xenograft nude mice, at least in part, by inhibiting the pro-apoptotic activity of cisplatin.

\section{Materials and methods}

Reagents and antibodies. LH was purchased from Merck KGaA (Darmstadt, Germany) and cisplatin was purchased from Selleck Chemicals LLC. (Houston, TX, USA). The antibodies used in the immunohistochemistry assay were anti-ki67 (cat. no. MA5-14520; Neomarkers, Inc., Waltham, MA, USA) and anti-cleaved caspase-3 (cat. no. 9661S; Cell Signaling Technology, Inc., Danvers, MA, USA). 
Cell lines and culture conditions. The highly metastatic human ovarian cancer cell lines, HeyA8 and SKOV3ip1, were used as described previously (19). Cells were purchased from the MD Anderson Characterized Cell Line Core Facility (Houston, TX, USA). Cells were cultured in RPMI 1640 medium supplemented with $10 \%$ fetal bovine serum (both purchased from Gibco; Thermo Fisher Scientific, Inc., Waltham, MA, USA) and $0.5 \%$ gentamicin, maintained on plastic and incubated at $37^{\circ} \mathrm{C}$ in a mixture of $5 \% \mathrm{CO}_{2}$ and $95 \%$ air. Tumor cells were free of pathogenic murine viruses and mycoplasma. The cells were maintained at $37^{\circ} \mathrm{C}$ in a mixture of $5 \% \mathrm{CO}_{2}$ and $95 \%$ air for $<10$ weeks following recovery from a frozen stock.

Animals. A total of 80 age-matched 8-10-week-old female athymic nude mice (NCr-nu) weighing 15.7-20.3 g were purchased from the Shanghai SLAC Laboratory Animal Co., Ltd. (Shanghai, China). The mice were housed under specific pathogen-free conditions at the animal facility of Shanghai Medical School, Fudan University (Shanghai, China). All mice were bred in a specific pathogen-free animal facility and were housed 4 per cage bedded with heat-treated chipped hardwood which was changed weekly. The facility used a $12 \mathrm{~h}$ light/dark cycle, and a standardized room temperature and humidity (30-70\%). Sterile pelleted food and water were freely available. Animal care and experiments were approved by the Institutional Animal Care and Use Committee at Shanghai Medical School, Fudan University and all procedures complied with the Guide for the Care and Use of Laboratory Animals published by the US National Institutes of Health (20).

Orthotopic tumor implantation and treatment. Sub-confluent cultures of HeyA8 and SKOV3ip1 cells were harvested and suspended in Hank's balanced salt solution medium (Invitrogen; Thermo Fisher Scientific, Inc., Waltham, MA, USA). Cell viability was determined to be $>95 \%$ using trypan blue exclusion (19). Suspended cells were intraperitoneally implanted into mice at a concentration of $2.5 \times 10^{5}$ cells $/ 0.2 \mathrm{ml}$ for HeyA8 cells or $1.0 \times 10^{6}$ cells $/ 0.2 \mathrm{ml}$ for SKOV3ip1 cells. The 40 mice were randomly divided into 4 groups ( $n=10 /$ group) 7 days following tumor implantation. One group, which served as a control, received injections of PBS (equal volume, intraperitoneal, once a week), one group received LH alone (3 U/day, subcutaneous), one group received cisplatin alone $(2.5 \mathrm{mg} / \mathrm{kg} /$ week, intraperitoneal) and one group received $\mathrm{LH}$ (3 U/day, subcutaneous) combined with cisplatin (2.5 mg/kg/week, intraperitoneal). It has been demonstrated that low-doses (3 U/day, subcutaneously) of FSH induce an improved effect compared with high-doses (10 U/day, subcutaneous) (21). Therefore, $3 \mathrm{U} /$ day $\mathrm{LH}$ was administered to mice undergoing treatment with LH in the present study.

During tumor progression, mice in the control and $\mathrm{LH}$ groups became weaker and moribund. These mice exhibited increased tumor load, decreased food consumption, decreased activity, and increased size of the ascites. Most mice exhibited some of these symptoms, however, mice in the control and LH groups exhibited the most severe symptoms. Seeing as mice in these two groups did not receive cisplatin chemotherapy, this was in line with expectations (18). When most mice in the control and LH groups presented with symptoms and became moribund, all the mice in the experiment were sacrificed immediately. HeyA8-implanted mice were sacrificed following 3 weeks treatment and SKOVip1-implanted mice were sacrificed following 5 weeks treatment. Prior to sacrifice, mice underwent inhalation anesthesia with $2 \%$ isoflurane (Ruiwode Lifescience Co. Shenzhen, China.). Following collection of $\sim 1 \mathrm{ml}$ blood via a cardiac puncture under anesthesia, mice were sacrificed. Body and tumor weight and the number of tumor nodules were recorded. Additionally, ascites were collected, blood was collected via cardiac puncture and all other samples were collected following sacrifice of mice on day 28 for HeyA8-implanted mice and on day 42 for SKOVip1-implanted mice.

Prior to immunohistochemical (IHC) staining, tumor tissues were fixed in formalin (cat. no. 50-00-0; Sinopharm Chemical Reagent Co., Ltd., Shanghai, China) at room temperature for $\geq 24 \mathrm{~h}$ and embedded in paraffin. Tissues were then cut into $4-\mu \mathrm{M}$ thick sections.

Measurement of serum LH levels. Blood samples were collected from the inferior vena cava and allowed to clot for $2 \mathrm{~h}$ at room temperature prior to centrifugation for $20 \mathrm{~min}$ at 2,000 x g. Sera were then collected for LHlevel measurements using a Luteinizing Hormone Human ELISA kit (cat. no. EHLH) (Thermo Fisher Scientific, Inc.).

IHC staining for ki67 and cleaved caspase-3. Paraffin sections were deparaffinized and rehydrated, then antigen retrieval was performed using EZ antigen retrieval 3 solution (BioGenex Laboratories, San Ramon, CA, USA). Sections were then blocked with $3 \%$ hydrogen peroxide in methanol and $4 \%$ fish gelatin at room temperature for $30 \mathrm{~min}$. Sections were then incubated with rabbit anti-ki67 (1:200) or rabbit anti-cleaved caspase-3 (1:100) overnight at $4^{\circ} \mathrm{C}$. Following washing with PBS, sections were incubated with horseradish peroxidase-conjugated goat anti-rabbit immunoglobulin G (cat. no. 111-005-045; dilution, 1:200; Jackson ImmunoResearch Laboratories, Inc., West Grove, PA, USA) for $60 \mathrm{~min}$ at room temperature. Sections were visualized with a 3,3'-diaminobenzidine kit (Vector Laboratories, Inc.), counterstained with hematoxylin at room temperature for $10 \mathrm{sec}$, dehydrated and mounted in Richard-Allan Scientific ${ }^{\mathrm{TM}}$ Cytoseal $^{\mathrm{TM}}$ XYL (Thermo Fisher Scientific, Inc.). Antibody staining in the tissue sections was observed using a light microscope (x40 magnification).

The count of ki67- or cleaved caspase-3-positive cells was independently performed by two experienced pathologists. Briefly, each entire slide was evaluated and five fields were randomly visualized at a magnification of x200. Subsequently, the average proportion of positively stained tumor cells was calculated based on the results from the five fields using ImageJ software (version 1.31; National Institutes of Health, Bethesda, MD, USA).

Statistical analysis. All data are presented as the mean \pm standard error of the mean. For in vivo therapy experiments, 10 mice were used in each group, which enabled the detection of a $50 \%$ reduction in tumor size $(B$ error $=0.2)$. Continuous variables were compared using two-tailed Student's t-tests (for 2 groups) or one-way analysis of variance followed by Tukey's test ( $>2$ groups) if the data were 
A

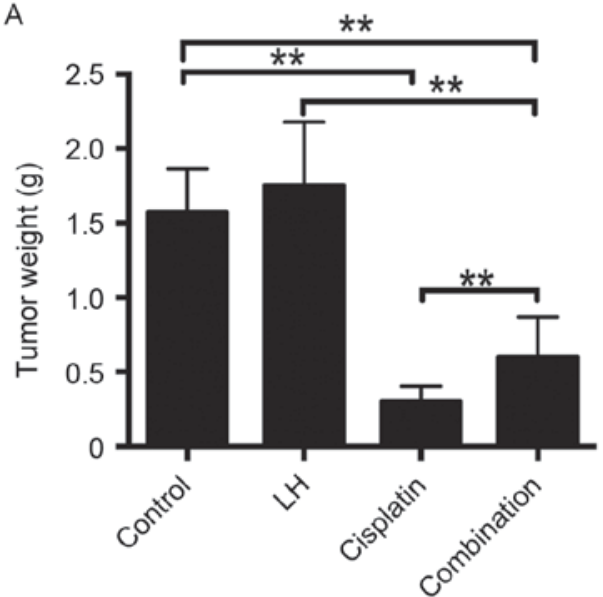

B

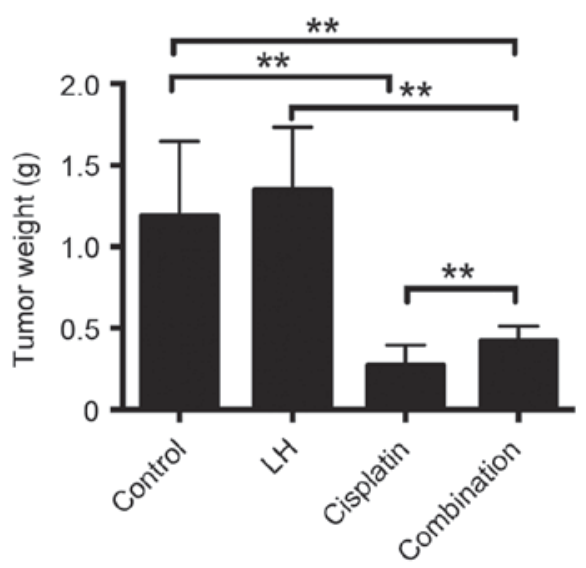

Figure 1. LH impaired the in vivo anti-tumor effect of cisplatin determined by tumor weight in (A) HeyA8-implanted and (B) SKOV3ip1-implanted nude mice. The data are presented as the mean \pm standard error of the mean; $n=10 ;{ }^{* *} \mathrm{P}<0.01$. LH, luteinizing hormone.

normally distributed. For non-parametric distributions, the Mann-Whitney U or the Kruskal-Wallis test were used. $\mathrm{P}<0.05$ was considered to indicate a statistically significant difference.

\section{Results}

LH impairs the in vivo anti-tumor effect of cisplatin. In the HeyA8- and SKOV3ip1- implanted mice, cisplatin alone but not LH alone treatment significantly reduced tumor weight and nodule number compared with the control group (Figs. 1 and 2). Although the treatment of cisplatin combined with LH still suppressed tumor weight and nodule number compared with the control group, the addition of LH significantly compromised the anti-tumor effect compared with cisplatin alone treatment (Figs. 1 and 2).

The maximum total tumor weight in SKOV3ip1-implanted nude mice was $2.0 \mathrm{~g}$ and in HeyA8-implanted nude mice, it was $2.4 \mathrm{~g}$. The maximum number of tumors in SKOV3ip1-implanted nude mice was 21 and that in HeyA8-implanted nude mice was 25 . The longest diameter of a single tumor was $\sim 1 \mathrm{~cm}$.

The volume of ascites may also represent the orthotopic tumor growth of ovarian cancer (22). The maximum volume of ascites observed in the current study was $12 \mathrm{ml}$. Treatment with cisplatin alone significantly reduced the volume of ascites compared with the control group in HeyA8- and SKOV3ip1-implanted nude mice (Fig. 3). Additionally, treatment with LH alone slightly increased the volume of ascites in HeyA8-implanted mice (Fig. 3A) and significantly increased the volume of ascites in SKOV3ip1-implanted mice (Fig. 3B). Combined treatment of cisplatin with LH significantly impaired the anti-tumor effect of cisplatin, indicated by the significant increase of ascite volume in the group receiving combination treatment compared with the group receiving treatment with cisplatin alone (Fig. 3). In addition, no differences were in mouse weights were observed among all four groups (Fig. 4). These results indicate that LH may impair the in vivo anti-tumor effect of cisplatin in nude mice implanted with epithelial ovarian cancer cells.

LH compromises the pro-apoptotic effect of cisplatin. The nuclear protein ki67 has been used as a biomarker for cell proliferation (23). Staining of proliferative and apoptotic cells and the quantitative analysis are depicted in Fig. 5. It was observed that the number of ki67-positive cells was significantly decreased in the cisplatin and combination treatment groups compared with the control group in SKOV3ip1-implanted mice (Fig. 5C). No significant differences were observed between the LH and control groups. Furthermore, combination treatment with cisplatin and $\mathrm{LH}$ resulted in increased ki67 expression compared to treatment with cisplatin alone (Fig. 5A and C), indicating that LH may compromise the anti-tumor activity of cisplatin.

Apoptosis is primary mechanism by which cisplatin induces cell death (24). Therefore, the current study investigated whether the impairment of cisplatin anti-tumor activity by LH results from the inhibition of $\mathrm{LH}$ on the cisplatin-mediated pro-apoptotic effect. The results of IHC staining demonstrated that combination treatment of cisplatin and LH led to fewer apoptotic cells determined by staining for cleaved caspase-3, compared with the group treated with cisplatin alone. However, combination treatment still induced more apoptosis than the control group (Fig. 5B and D). No significant differences were identified between the LH and control groups. These results indicate that $\mathrm{LH}$ impairs the in vivo anti-tumor effect of cisplatin, at least in part, by inhibiting the pro-apoptotic activity of cisplatin.

Serum levels of $\mathrm{LH}$ in the xenografted nude mice. In order to confirm the validity of the LH injection, the serum levels of LH were measured. As expected, LH was undetectable in the sera of control and cisplatin-treated mice, whereas the concentration of $\mathrm{LH}$ in the mouse sera of the groups treated with LH alone or LH combined with cisplatin was 30 U/1 in the HeyA8- and SKOV3ip1-implanted mice (Fig. 6).

\section{Discussion}

Ovarian cancer often occurs in postmenopausal women and is characterized by high gonadotropin levels ( $40 \mathrm{U} / 1)$. Therefore, gonadotropins including FSH and LH have been regarded as probable risk factors for the development of ovarian cancer. FSH has been identified to serve a function in the development 

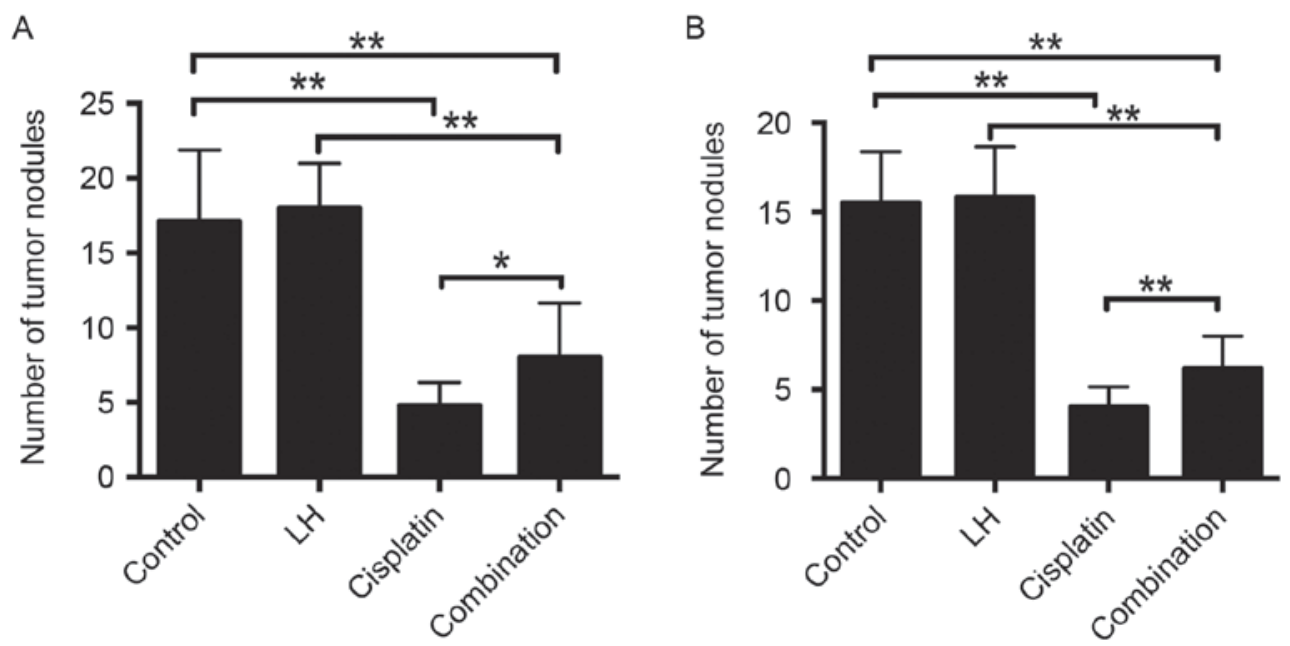

Figure 2. LH impaired in vivo anti-tumor effect of cisplatin determined by the number of tumor nodules in (A) HeyA8-implanted and (B) SKOV3ip1-implanted nude mice. The data are presented as the mean \pm standard error of the mean; $n=10$; ${ }^{*} \mathrm{P}<0.05$ and ${ }^{* *} \mathrm{P}<0.01$. LH, luteinizing hormone.
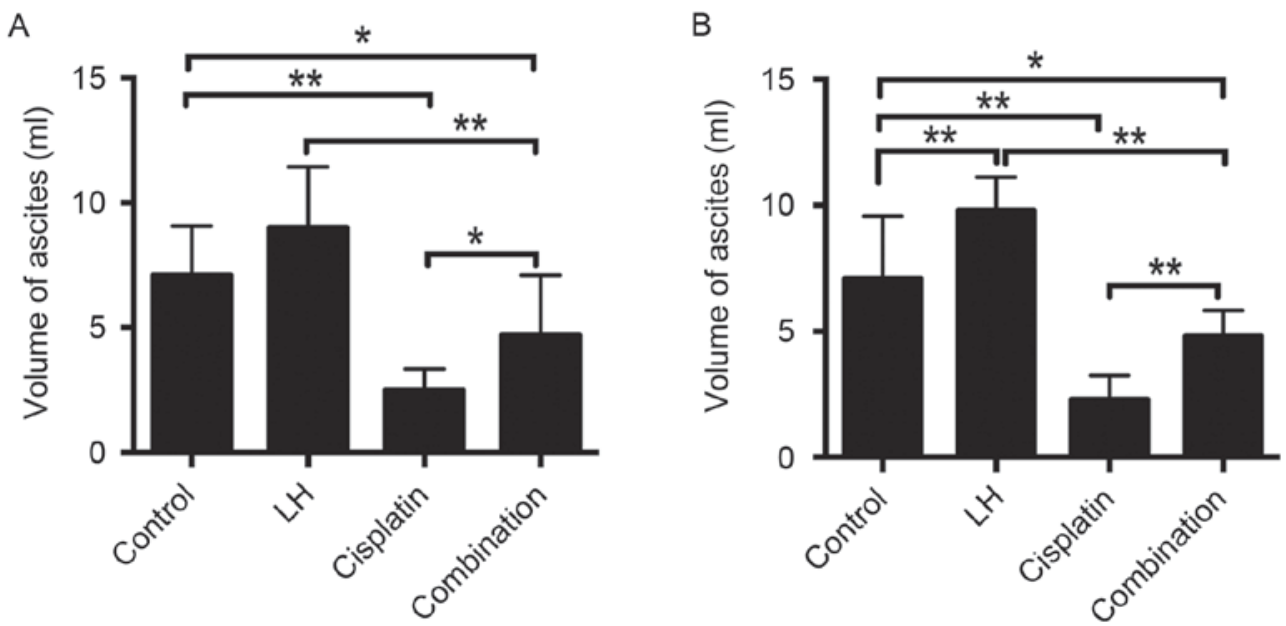

Figure 3. LH impaired in vivo anti-tumor effect of cisplatin determined by ascite volume in (A) HeyA8-implanted and (B) SKOV3ip1-implanted nude mice. The data are presented as the mean \pm standard error of the mean; $n=10$; ${ }^{*} \mathrm{P}<0.05$ and ${ }^{* * *} \mathrm{P}<0.01$. LH, luteinizing hormone.

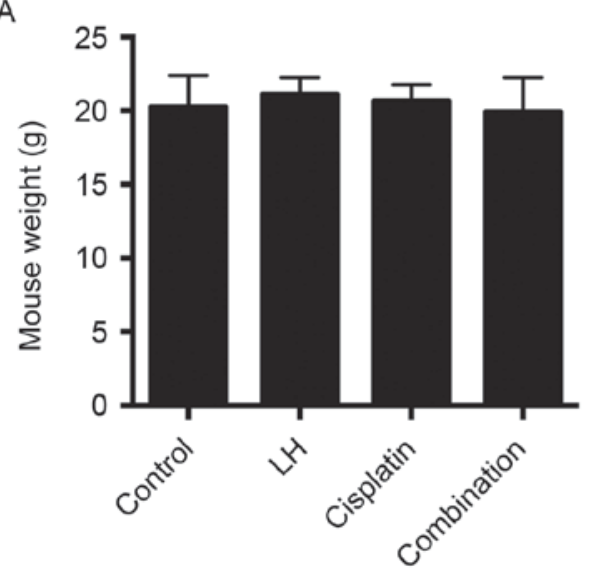

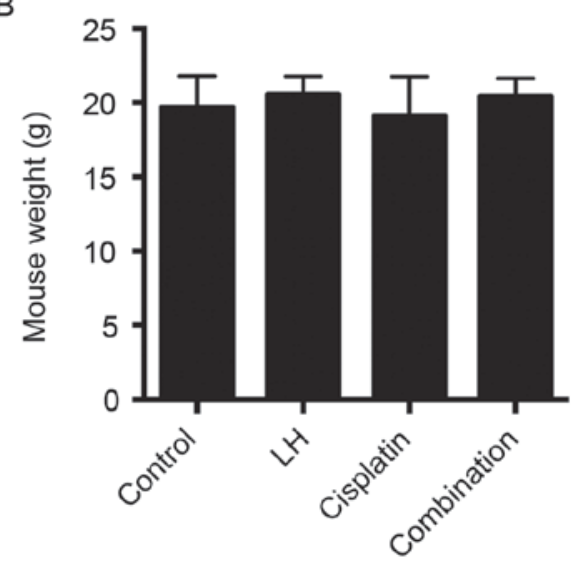

Figure 4. Treatment with cisplatin alone, LH alone, or cisplatin combined with LH did not affect the weights of (A) HeyA8-implanted and (B) SKOV3ip1-implanted nude mice. The data are presented as the mean \pm standard error of the mean; $\mathrm{n}=10$. LH, luteinizing hormone.

and progression of ovarian cancer in vitro and in vivo $(21,25)$. However, previous studies have identified that LH is able to inhibit apoptosis and facilitate angiogenesis in vitro (26-28), and therefore the effect of $\mathrm{LH}$ in vivo is worth investigating. 
A

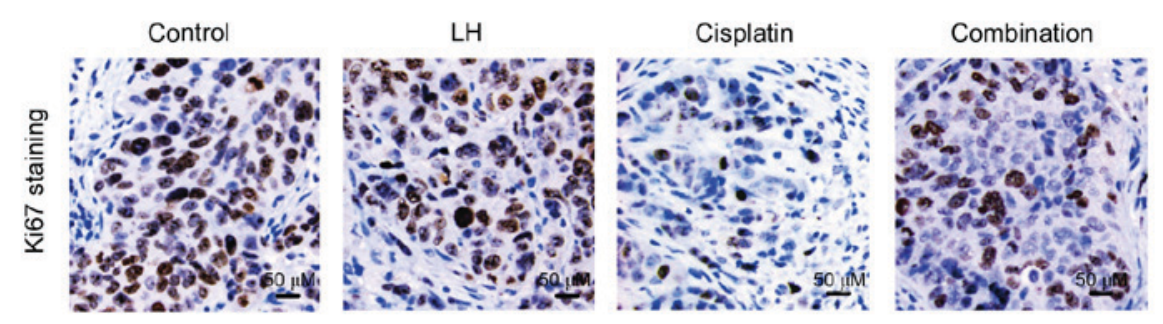

B

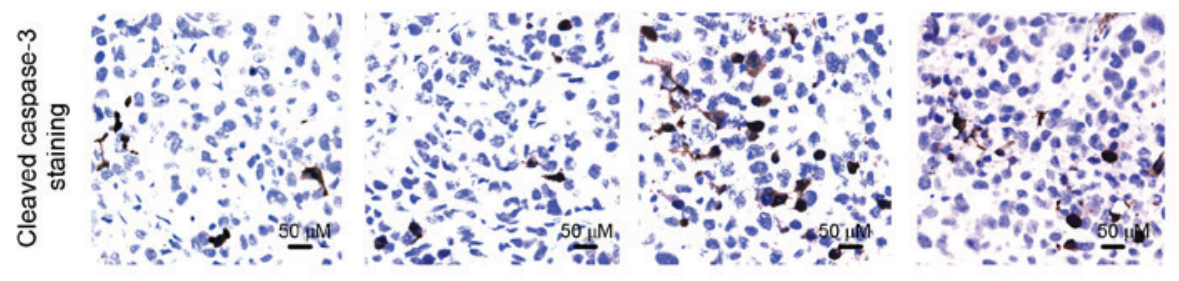

$\mathrm{C}$

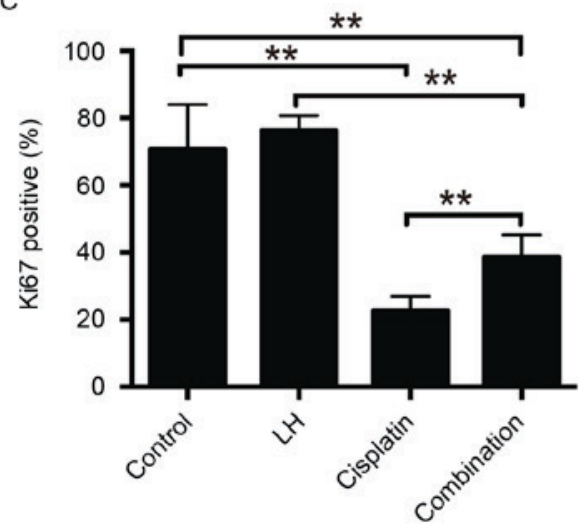

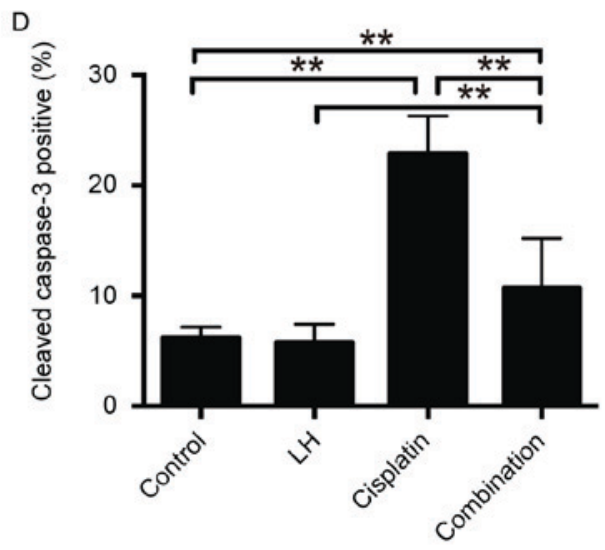

Figure 5. Proliferating cells stained by (A) ki67 and apoptotic cells stained by (B) cleaved caspase-3 with their respective quantitative analyses (C and D). The data are presented as the mean \pm standard error of the mean; $n=10 ;{ }^{* *} \mathrm{P}<0.01$. Scale bar $=50 \mu \mathrm{m}$. LH, luteinizing hormone.
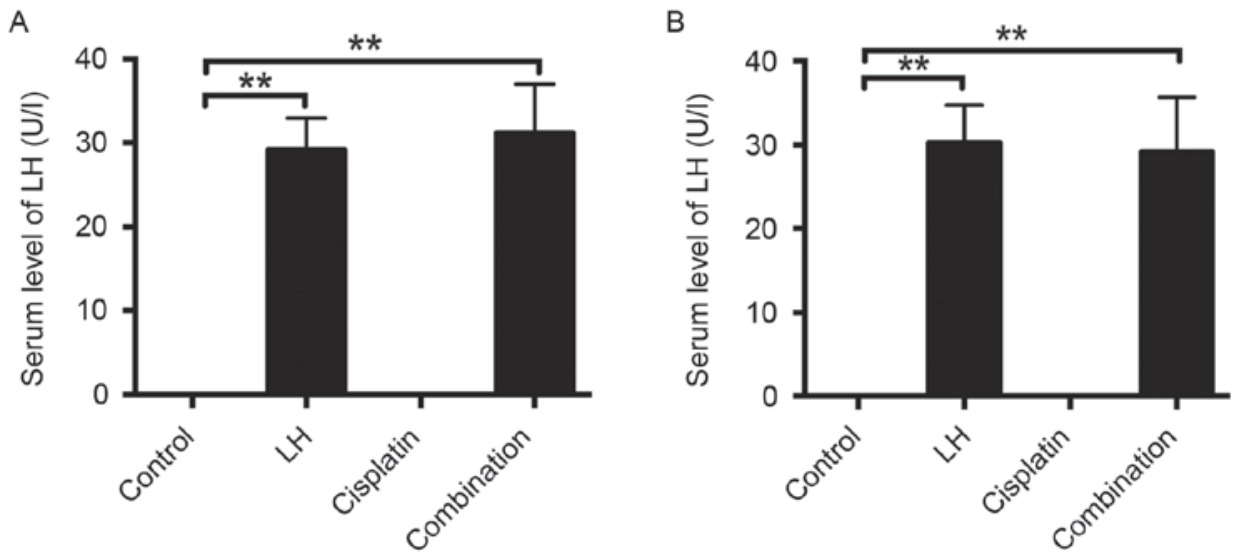

Figure 6. Serum levels of LH in (A) HeyA8-implanted or (B) SKOV3ip1-implanted nude mice measured by ELISA. The data are presented as the mean \pm standard error of the mean; $\mathrm{n}=10 ;{ }^{*} \mathrm{P}<0.01$. $\mathrm{LH}$, luteinizing hormone.

In the present study, two epithelial ovarian cancer cell lines, HeyA8 and SKOV3ip1, were implanted into nude mice and the effect of exogenous LH was detected on the cisplatin anti-tumor activity. ELISA was employed to verify the LH serum level $\sim 30 \mathrm{U} / 1$ in mice treated with $\mathrm{LH}$, which is comparable to the LH level in patients with post-menopausal ovarian cancer (29). Cisplatin is capable of inducing apoptosis in ovarian cancer cells $(30,31)$. In the present study, cisplatin significantly inhibited the growth of ovarian tumors, number of tumor nodules and volume of ascites. In addition, it was revealed that treatment with $\mathrm{LH}$ alone exhibited a minimal effect on tumor weights and the number of tumor nodules, although it increased the volumes of ascites. The in vivo anti-tumor effect of cisplatin was significantly impaired when 
administered in combination with LH, which was determined by the increase of growth of ovarian tumors, the number of tumor nodules and the volume of ascites compared with the group that underwent treatment with cisplatin alone.

It was observed that the number of apoptotic cells with cleaved caspase-3-positive was significantly reduced following combination treatment with $\mathrm{LH}$ and cisplatin, and that the number of proliferative cells with ki67-positive was increased compared with the group that received treatment with cisplatin alone. However, the present study did not investigate the underlying antitumor mechanisms responsible for the effect of LH on impairing cisplatin in vivo.

Collectively, results of the present study indicate that LH weakens the anti-tumor effect of cisplatin in vivo, and LH may contribute to the development of drug resistance to cisplatin in ovarian cancer. LH-antagonists may be used in the near future to reverse the effect of $\mathrm{LH}$ and to aid in the treatment of ovarian cancer (32).

\section{References}

1. Siegel RL, Miller KD and Jemal A: Cancer statistics, 2016. CA Cancer J Clin 66: 7-30, 2016.

2. Stadel BV: Letter: The etiology and prevention of ovarian cancer. Am J Obstet Gynecol 123: 772-774, 1975.

3. Mertens-Walker I, Baxter RC and Marsh DJ: Gonadotropin signalling in epithelial ovarian cancer. Cancer Lett 324: 152-159, 2012.

4. Venn A, Watson L, Bruinsma F, Giles G and Healy D: Risk of cancer after use of fertility drugs with in-vitro fertilisation. Lancet 354: 1586-1590, 1999.

5. Brekelmans CT: Risk factors and risk reduction of breast and ovarian cancer. Curr Opin Obstet Gynecol 15: 63-68, 2003.

6. Holschneider CH and Berek JS: Ovarian cancer: Epidemiology, biology, and prognostic factors. Semin Surg Oncol 19: 3-10, 2000.

7. Tavani A, Ricci E, La Vecchia C, Surace M, Benzi G, Parazzini F and Franceschi S: Influence of menstrual and reproductive factors on ovarian cancer risk in women with and without family history of breast or ovarian cancer. Int J Epidemiol 29: 799-802, 2000.

8. Daly M and Obrams GI: Epidemiology and risk assessment for ovarian cancer. Semin Oncol 25: 255-264, 1998.

9. Gnagy S, Ming EE, Devesa SS, Hartge P and Whittemore AS: Declining ovarian cancer rates in U.S. women in relation to parity and oral contraceptive use. Epidemiology 11: 102-105, 2000.

10. Zheng W, Lu JJ, Luo F, Zheng Y, Feng Yj, Felix JC, Lauchlan SC and Pike MC: Ovarian epithelial tumor growth promotion by follicle-stimulating hormone and inhibition of the effect by luteinizing hormone. Gynecol Oncol 76: 80-88, 2000.

11. Kurbacher CM, Jäger W, Kurbacher JA, Bittl A, Wildt L and Lang N: Influence of human luteinizing hormone on cell growth and CA 125 secretion of primary epithelial ovarian carcinomas in vitro. Tumour Biol 16: 374-384, 1995.

12. Kuroda H, Mandai M, Konishi I, Yura Y, Tsuruta Y, Hamid AA, Nanbu K, Matsushita K and Mori T: Human chorionic gonadotropin (hCG) inhibits cisplatin-induced apoptosis in ovarian cancer cells: Possible role of up-regulation of insulin-like growth factor-1 by hCG. Int J Cancer 76: 571-578, 1998.

13. Tashiro H, Miyazaki K, Okamura H, Iwai A and Fukumoto M: c-myc over-expression in human primary ovarian tumours: Its relevance to tumour progression. Int J Cancer 50: 828-833, 1992

14. Tourgeman DE, Lu JJ, Boostanfar R, Amezcua C, Felix JC and Paulson RJ: Human chorionic gonadotropin suppresses ovarian epithelial neoplastic cell proliferation in vitro. Fertil Steril 78: 1096-1099, 2002.
15. Choi JH, Choi KC, Auersperg N and Leung PC: Gonadotropins activate proteolysis and increase invasion through protein kinase A and phosphatidylinositol 3-kinase pathways in human epithelial ovarian cancer cells. Cancer Res 66: 3912-3920, 2006.

16. Tilly JL, Tilly KI, Kenton ML and Johnson AL: Expression of members of the bcl-2 gene family in the immature rat ovary: Equine chorionic gonadotropin-mediated inhibition of granulosa cell apoptosis is associated with decreased bax and constitutive bcl-2 and bcl-xlong messenger ribonucleic acid levels. Endocrinology 136: 232-241, 1995.

17. Galluzzi L, Senovilla L, Vitale I, Michels J, Martins I, Kepp O, Castedo M and Kroemer G: Molecular mechanisms of cisplatin resistance. Oncogene 31: 1869-1883, 2012.

18. Xia L, Wen H, Han X, Tang J and Huang Y: Luteinizing hormone inhibits cisplatin-induced apoptosis in human epithelial ovarian cancer cells. Oncol Lett 11: 1943-1947, 2016.

19. Lu C, Shahzad MM, Moreno-Smith M, Lin YG, Jennings NB, Alle JK, Landen CN, Mangala LS, Armaiz-Pena GN, Schmandt R, et al: Targeting pericytes with a PDGF-B aptamer in human ovarian carcinoma models. Cancer Biol Ther 9: $176-182,2010$.

20. Guide for the Care and Use of Laboratory Animals: National Research Council (US) Committee for the update of the guide for the care and use of laboratory animals. 8th edition. Washington (DC): National Academies Press (US), 2011.

21. Huang Y, Jin H, Liu Y, Zhou J, Ding J, Cheng KW, Yu Y and Feng Y: FSH inhibits ovarian cancer cell apoptosis by up-regulating survivin and down-regulating PDCD6 and DR5. Endocr Relat Cancer 18: 13-26, 2010.

22. Garofalo A, Naumova E, Manenti L, Ghilardi C, Ghisleni G, Caniatti M, Colombo T, Cherrington JM, Scanziani E, Nicoletti MI and Giavazzi R: The combination of the tyrosine kinase receptor inhibitor SU6668 with paclitaxel affects ascites formation and tumor spread in ovarian carcinoma xenografts growing orthotopically. Clin Cancer Res 9: 3476-3485, 2003.

23. Scholzen T and Gerdes J: The Ki-67 protein: From the known and the unknown. J Cell Physiol 182: 311-322, 2000.

24. Fisher DE: Apoptosis in cancer therapy: Crossing the threshold. Cell 78: 539-542, 1994.

25. Huang Y, Hua K, Zhou X, Jin H, Chen X, Lu X, Yu Y, Zha X and Feng Y: Activation of the PI3K/AKT pathway mediates FSH-stimulated VEGF expression in ovarian serous cystadenocarcinoma. Cell Res 18: 780-791, 2008.

26. Zhang Z, Liao H, Chen X, Zheng Y, Liu Y, Tao X, Gu C, Dong L, Duan T, Yang Y, et al: Luteinizing hormone upregulates survivin and inhibits apoptosis in ovarian epithelial tumors. Eur J Obstet Gynecol Reprod Biol 155: 69-74, 2011.

27. Xia L, Wen H, Han X, Tang J and Huang Y: Luteinizing hormone inhibits cisplatin-induced apoptosis in human epithelial ovarian cancer cells. Oncol Lett 11: 1943-1947, 2016.

28. Liao H, Zhou Q, Gu Y, Duan T and Feng Y: Luteinizing hormone facilitates angiogenesis in ovarian epithelial tumor cells and metformin inhibits the effect through the mTOR signaling pathway. Oncol Rep 27: 1873-1878, 2012.

29. Rzepka-Górska I, Chudecka-Głaz A and Kosmowska B: FSH and LH serum/tumor fluid ratios and malignant tumors of the ovary. Endocr Relat Cancer 11: 315-321, 2004.

30. Kim JH, Jeong SJ, Kim B, Yun SM, Choi DY and Kim SH: Melatonin synergistically enhances cisplatin-induced apoptosis via the dephosphorylation of ERK/p90 ribosomal S6 kinase/heat shock protein 27 in SK-OV-3 cells. J Pineal Res 52: 244-252, 2012.

31. Mabuchi S, Altomare DA, Cheung M, Zhang L, Poulikakos PI, Hensley HH, Schilder RJ, Ozols RF and Testa JR: RAD001 inhibits human ovarian cancer cell proliferation, enhances cisplatin-induced apoptosis, and prolongs survival in an ovarian cancer model. Clin Cancer Res 13: 4261-4270, 2007.

32. Tan O and Bukulmez O: Biochemistry, molecular biology and cell biology of gonadotropin-releasing hormone antagonists. Curr Opin Obstet Gynecol 23: 238-244, 2011. 